\title{
Redshift measurement through star formation
}

\author{
Mikkel O. Lindholmer and Kevin A. Pimbblet
}

\author{
E. A. Milne Centre for Astrophysics, University of Hull, Cottingham Rd, Hull HU6 7RX, UK \\ e-mail: M.Lindholmer@2016.hull.ac.uk
}

Received 19 March 2018 / Accepted 15 December 2018

\begin{abstract}
In this work we use the property that, on average, star formation rate increases with redshift for objects with the same mass - the so called galaxy main sequence - to measure the redshift of galaxy clusters. We use the fact that the general galaxy population forms both a quenched and a star-forming sequence, and we locate these ridges in the SFR- $M_{\star}$ plane with galaxies taken from the Sloan Digital Sky Survey in discrete redshift bins. We fitted the evolution of the galaxy main sequence with redshift using a new method and then subsequently apply our method to a suite of X-ray selected galaxy clusters in an attempt to create a new distance measurement to clusters based on their galaxy main sequence. We demonstrate that although it is possible in several galaxy clusters to measure the main sequences, the derived distance and redshift from our galaxy main sequence fitting technique has an accuracy of $\sigma_{z}= \pm 0.017 \cdot(z+1)$ and is only accurate up to $z \approx 0.2$.
\end{abstract}

Key words. methods: observational - galaxies: clusters: general

\section{Introduction}

The main sequence of galaxies is a relationship between the star formation rate (SFR) or specific star formation rate (sSFR) relative to its stellar mass $\left(M_{\star}\right)$. The main sequence has been observed at both low and high redshift, using deep and (or) wide-field surveys (e.g., Guzmán et al. 1997; Bell et al. 2005; Reddy et al. 2006; Salim et al. 2007; Daddi et al. 2007; Noeske et al. 2007a; Elbaz et al. 2007; Rodighiero et al. 2011; Wuyts et al. 2011; Salmi et al. 2012; Whitaker et al. 2012; Guo et al. 2013, 2015; Pannella et al. 2015; amongst others) and has received attention by simulators in recent years (e.g., Sparre et al. 2016; Obreja et al. 2014; Dutton et al. 2010; Bouche et al. 2010).

Noeske et al. (2007a) presents one of the most comprehensive reviews of the main sequence of galaxies. They use data from the All-Wavelength Extended Groth Strip International Survey (AEGIS; Davis et al. 2007) to show that despite some scatter in SFR $-M_{\star}$ space, star-forming galaxies form a relationship with less active red sequence galaxies lying below, although these are likely to be active galactic nuclei (AGN) in origin (Weiner et al. 2007). Noeske et al. (2007a) notes that the slope of this relationship tends to flatten out at higher redshift, which could be due to completeness issues in AEGIS. At lower redshift, the scatter in the relationship is constant out to at least $z \approx 1.0$ (see also Speagle et al. 2014). It should be noted that the galaxy main sequence evolves in redshift, such that at higher redshift the main sequence has a higher SFR. This is not due to the outer envelope of the relationship changing; it is the actual relationship itself that translates in the SFR- $M_{\star}$ plane (see also Zamojski et al. 2007).

It has been observed that the galaxy main sequence is a relationship that has a multi-wavelength appearance, from the optical through the infra-red and radio. It is argued (cf. Pannella et al. 2015; Tasca et al. 2015; Rodighiero et al. 2014; Karim et al. 2011; Elbaz et al. 2011; Brinchmann et al.
2004; Noeske et al. 2007b) that the reason behind the relationship between redshift and SFR $-M_{\star}$ can be attributed to a staged galaxy formation, which is an expression of the widely discussed downsizing phenomena (e.g. Cowie et al. 1996; Brinchmann \& Ellis 2000; Heavens et al. 2004; Thomas et al. 2005; Bundy et al. 2006; Bell et al. 2005; Fontanot et al. 2009; Eliche-Moral et al. 2010).

In simple terms, the argument is that as galaxies have decreasingly less mass they are likely to have their major star formation (SF) episodes occur at decreasing redshift.

More recent work has focussed on both the intrinsic scatter of the star-forming galaxies in the main sequence, as well as the issue of whether there is any evolution in the slope of the relationship over time.

Turning first to the scatter, Noeske et al. (2007a) find an intrinsic scatter of around $0.35 \mathrm{dex}$ in SFR about the main sequence that includes $34 \%$ of the galaxy population. Several figures for the scatter of the main sequence reported elsewhere show that this value is typical for a wide variety of redshift ranges (cf. Rodighiero et al. 2011; Whitaker et al. 2012, but see also Guo et al. 2013). However, it should be noted that there have been reports that the scatter can increase with higher galaxy stellar mass. Guo et al. (2015) show that higher mass galaxies at low redshift $(z<0.03)$ have more scatter in their SSFR than lower mass ones do.

The slope of the main sequence appears to be constant over both galaxy stellar mass and redshift (see Karim et al. 2011), as long as the selection effects are taken into account (Guo et al. 2013). Guo et al. (2013) make it clear that selection effects have a large impact on the evolution of the slope in regards to redshift. Once this is taken care of it has been reported several times that the slope is fixed at about unity out to high redshifts in the SFR $-M_{\star}$ plane (e.g. Elbaz et al. 2007; Noeske et al. 2007a; Daddi et al. 2007; Gilbank et al. 2011; Salmi et al. 2012).

We predict a number of significant uses of the galaxy main sequence. For example, with greater precision we could use it as 
an extra check on redshift for galaxy cluster in cases where measuring redshift is not achievable, for example due to a paucity of spectroscopy. We can additionally use it to identify other atypical clusters like the Coma cluster (Pimbblet et al. 2014), and potentially further explore the cause of their deviation from SFR expectation values.

The plan of this work is as follows. In Sect. 2 we present the data used for both finding clusters and getting the SFR and mass of each of the member galaxies. In Sect. 3 we go through our method of analysing the galaxy main sequence and its evolution in redshift. In Sect. 4 we present our results and test how precise the method is. In Sect. 5 we conclude our findings and discuss the implications and uses of them. Throughout this work we use the Spergel et al. (2007) $\Lambda$ CDM cosmology in which $\Omega_{\mathrm{M}}=0.238, \Omega_{\Lambda}=0.762$, and $H_{0}=73 \mathrm{~km} \mathrm{~s}^{-1} \mathrm{Mpc}^{-1}$.

\section{Data}

To find the general relationship between redshift and SFR- $M_{\star}$ plane of the main sequence (Sect. 3) we use Sloan Digital Sky Survey (SDSS) data release 8 (DR8), as it is the latest available data release where all the masses and SFR have been calculated homogeneously (Brinchmann et al. 2004 and Kauffmann et al. 2004). The data are split into bins of $z=0.001$ in width, up to $z=0.20$, and analysed separately. Hence each of these bins are complete to the spectroscopic limit and provide a stellar mass limit that is approximately constant within any given bin, although clearly the stellar mass limit and completeness does vary significantly from bin to bin over the redshift range probed. Since SFR and $M_{\star}$ are a key part of the process, we only use galaxies that have those values available. Importantly, we note that the galaxy main sequences should have the same locus in both the discrete redshift bins and any given galaxy cluster.

The cluster sample used in this work (Sect. 4) is selected from the X-Ray Clusters Database. We select clusters with luminosity $L_{\mathrm{X}}>0.3 \times 10^{44} \mathrm{erg} \mathrm{s}^{-1}$ and within a redshift range 0.0-0.20. Additionally, Base de Données Amas de Galaxies X (BAX) is used to identify the rough X-ray centre of the galaxy cluster centre and therefore the corresponding galaxies in SDSS DR8 that are potentially part of the cluster.

To determine cluster membership, we used the method outlined in Diaferio (1999) to identify which galaxies and substructures are part of these clusters. We imposed a minimum limit of 50 galaxies per cluster to ensure we have sufficient galaxies to work with. This gives us a total of 129 clusters for which we find more than 50 galaxy entries in SDSS DR8 although a significant number of them are so messy in the SFR $-M_{\star}$ plane that our method either does not detect the ridges or the detection we make has an standard deviation of above $0.05 \log \left(M_{\odot}\right.$ year $\left.^{-1}\right)$ SFR.

In our analysis, we aim to measure both the star-forming sequence of galaxies, as well as the quenched sequence on the SFR $-M_{\star}$ plane where possible. Therefore for the redshifts that we examine, we need to ensure that the data and their completeness enable us to measure both. We did this by creating log histograms of sSFR for the redshift bins. We fitted a line to the linear portion of the log histogram and extrapolated to determine where the completeness drops below an acceptable level. We removed all galaxies that are part of those low completeness bins from our analysis. This sometimes results in part of the quenched ridge being completely eradicated, and in those cases we acknowledge it is not possible to locate the quenched ridge. In most cases, however, we were able to fit both the star-forming ridge and the quenched ridge, or a sufficient part of the quenched ridge, as desired.
Even where some of the completeness issues affect the starforming ridge, the star-forming ridge can be fit and the number of galaxies removed from this ridge due to completeness is inconsequential to the fit performed.

Upon checking the resulting data after cuts, we see that some of our data are slightly under SFR $=0.1$, which seems to align fairly well with SDSS data used by others (see Schreiber et al. 2014; Renzini \& Peng 2015), as well as data from other sources (Davies et al. 2016).

\section{Method}

The outline of our method is first to find an estimate of the inclination of the ridges of the SFR $-M_{\star}$ plane. We then more precisely identify the location of the ridge and translate that into a "standardized" SFR. The standardized SFR is then applied to ridges found in the galaxy cluster sample to determine the distance to them. We use the same method for both analysing redshift bins and subsequently the galaxy clusters. The only difference comes from scaling depending on the number of galaxies in the observed bin or cluster. We can do this since we can assume that the galaxies in galaxy clusters are, in general, a good representation of the redshift bin in which they are located.

\subsection{Inclination}

To determine a rough inclination we start by sampling the over density of all the areas in the SFR- $M_{\star}$ plane. We then assume that the point with the largest over density should be on or close to the top of the ridge that represents the galaxy main sequence. After this we fit a line through that point and the largest densities in the SFR $-M_{\star}$ plane as seen in Fig. 1, where the highest density point is located in the star-forming ridge. This gives a rough estimate of the inclination of the galaxy main sequence that is then used as an initial guess for fitting the galaxy main sequence.

Using the initial guess of the inclination we then place angled lines across the galaxy main sequence and measure the density along the lines with the ultimate goal of determining a double Gaussian fit along them. These lines are used to measure the exact location of the ridge and therefore need to be close to perpendicular to the ridge. However since the range of observed values is far larger for SFR than it is for $M_{\star}$ using a perpendicular line to the galaxy main sequence would not be correct. As such we correct for this by adjusting the lines to be steeper in SFR.

\subsection{Fitting}

We then measure the density along the lines. This gives us a rough double Gaussian curve where the centres of the Gaussians are located at the quenched sequence and the star-forming sequence (see Fig. 2). Besides these regions most of the other galaxies are located in the transition region, the so-called "green valley". Sometimes it is not possible to get a clean double Gaussian fit due to completeness removing the quenched ridge along the line we are fitting, or if the star-forming ridge is sparse, or if the green valley has a significant artefact along the line we are measuring. In such cases we remove the Gaussian with the largest amplitude, since it is the most significant one and then remeasure with a new double Gaussian where we allow the fit to reduce one of the Gaussians to zero amplitude. One Gaussian will then represent the remaining ridge while the remaining noise should be hard to fit to any shape. A poor model of the noise is 


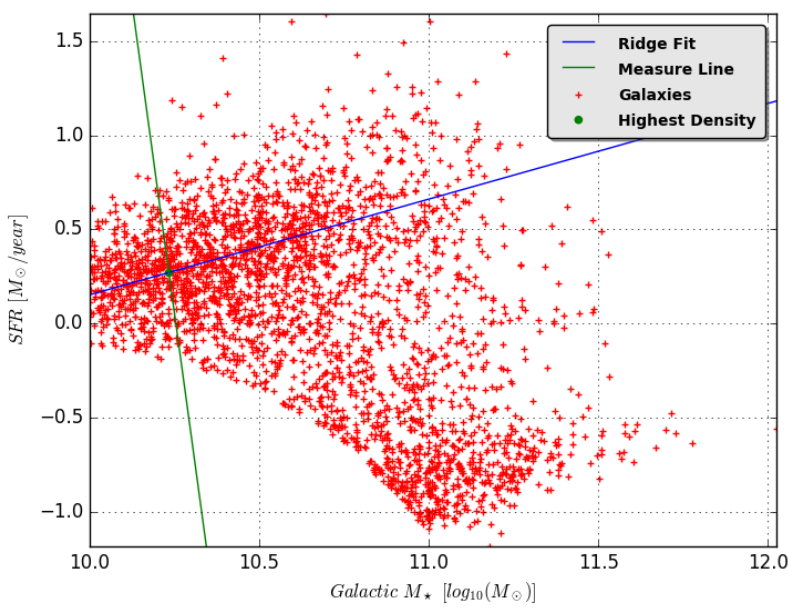

Fig. 1. All the galaxies where SFR and mass have been measured in the redshift bin $0.092<z<0.093$. The red crosses are individual galaxies while the green circle shows the point of highest density and the blue line shows our rough fit of the density ridge. It can clearly be seen that there is an overdensity along the solid blue line. By inspection it can also be seen that there is another overdensity around -0.8 SFR ranging from $10^{10.8} M_{\odot}$ to about $10^{11.1} M_{\odot}$. We make 199 lines that are parallel to the green one and measure overdensities along them to identify where the centre of the ridge is. By combining all these 200 measurements we can get a good idea of where the ridges are. The angle of the green line to the ridge has been optimized such that we have the smallest variance across all the redshift bins.

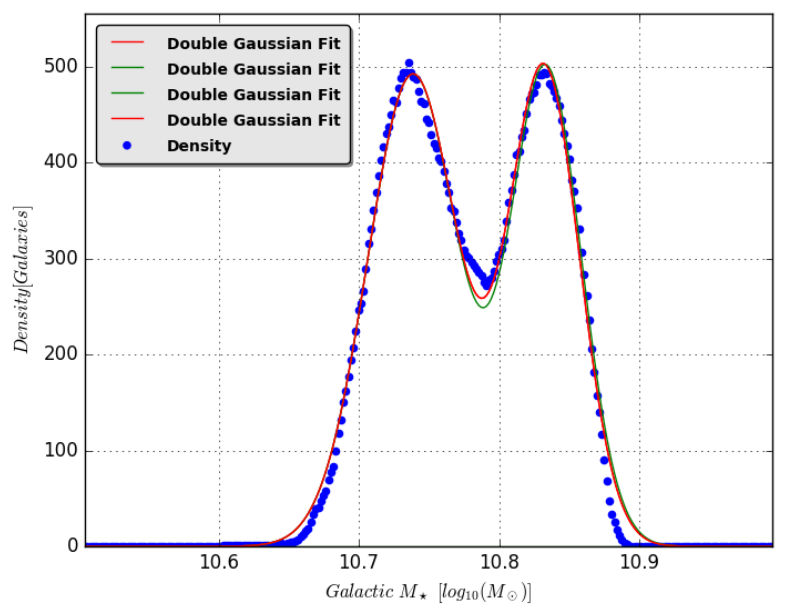

Fig. 2. Example of how we fit at one point in a ridge. The blue dots represent the density around each point measured across the ridge (see the green line in Fig. 1) and the red line is our fit. The quenched ridge top is located around 10.84 and our fit to it is well within accepted errors. The star-forming ridge top is located at around 10.72 and is also well within our accepted range. The green valley is not fit very well in this plot, but this is not important as we are not trying to measure anything in that area.

acceptable since this is meant to detect what part of our measurement is the remaining ridge and the ridge should appear roughly as a Gaussian. We then do a test to check the likelihood that we actually found a ridge and remove any measurements with a signal to noise ratio that is too low.

By then plotting the centre of each of the Gaussian fits in the SFR $-M_{\star}$ plane we can then see a pattern emerging in the form of one or two lines depending on how well the star-forming sequence has been fitted (Fig. 3). By fitting a line to one of these sequence points we then arrive at a fit for the ridge.

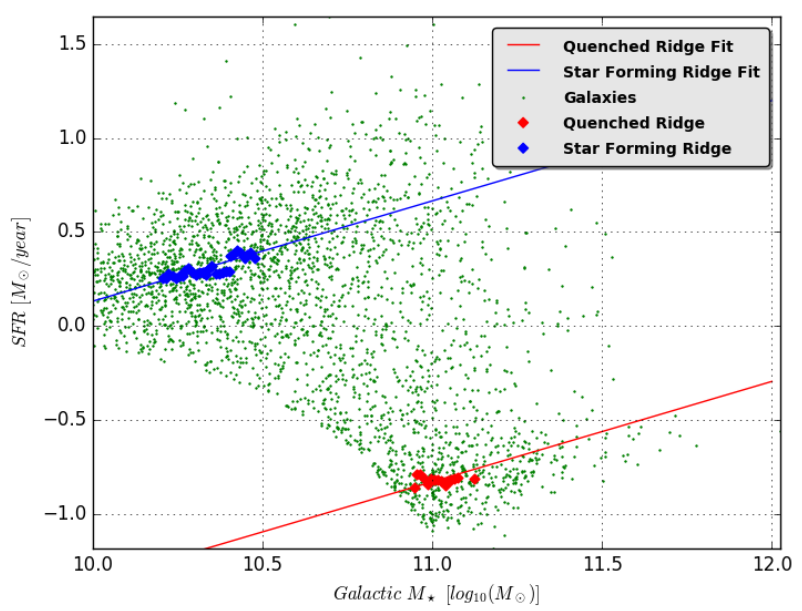

Fig. 3. Fit to the quenched ridge along the red line and the star-forming ridge along the blue line. The diamonds are the locations the Gaussian fits have indicated. The fits for the star-forming ridge line up very well whereas the fits for the quenched sequence only line up for a small section and have considerable scatter. This is mainly since most of the ridge has been removed due to completeness concerns so we are only measuring at the very high mass end of the ridge where it is likely to be more noisy.

\subsection{Ridge fitting}

For the initial fits of the ridges we have freely fitted the inclination of the ridge. This creates some scatter in the inclination we have fitted due to uncertainties and selection criteria, especially at lower redshift. When analysing the scatter at low redshift it is obvious that it is mainly towards higher inclination. This is because there are few galaxies far below the quenched sequence and the area around $10^{10} M_{\odot}$ has the largest number of galaxies, causing the ridge to typically be fitted better in that area. To avoid this systematic error we only use the area where we see little scatter in the inclination (redshift $0.1<z<0.18$ ) to give us an expected value for the inclination for all of our data. We find that this value is constant as suggested by Guo et al. (2013). As further demonstrated by Guo et al. (2013) we can expect this to also be true for lower redshift and we therefore assume that it works there as well.

To measure the position of the ridge in the SFR $-M_{\star}$, plane we use a standardized measure of the SFR. This is simply done by calculating the SFR of our ridge fit at the $10^{10} M_{\odot}$ point. Doing this allows us to get a measure of how the galaxy main sequence evolves in redshift. By using the same inclination for all the data we arrive at a relationship in SFR-redshift space with very little scatter. We then fit this relationship to a straight line, using two free parameters. This can then be used as an expected value to compare to standardized measures of cluster SFR.

\section{Results and discussion}

In this section we will go over the results we get from the method when comparing our results obtained from redshift bins to the galaxy clusters. We will first present the fit to the redshift bin ridges to ensure that they are good and we highlight the few locations which are less than optimal. After this, we present the evolution of the ridges in redshift and make a fit to this evolution. Finally, we try to apply this method to a sample of clusters to test the method's viability in measuring the redshift of the clusters. 


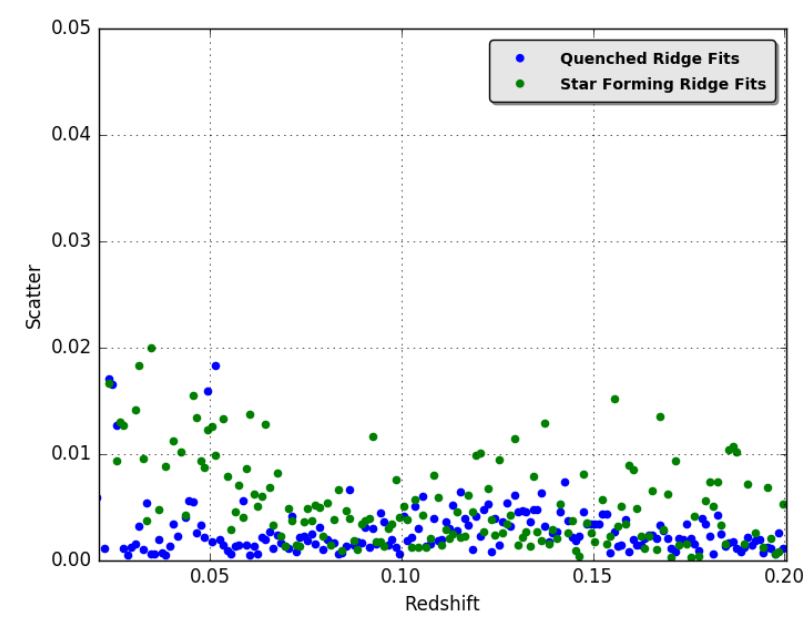

Fig. 4. Standard deviation of the fit of the ridges in each of the redshift bins. At redshifts of $z<0.021$, the method does not detect any ridges due to the number of galaxies in the bins. We also see increasing irregularity in the ridges below about redshift $z<0.05$. Besides this we the star-forming ridge having very high dispersion at redshifts above $z<0.15$ due to being very sparse at these redshifts.

\subsection{Ridge fitting}

Since we are trying to create a general method to use for many different sets of galaxies at different redshifts, we have decided to use a single set of parameters across all the bins. By doing so we retain a generally low dispersion in the fits as it makes sure that there are no systematic errors due to using different parameters. As can be seen in Fig. 4, we mostly obtain good fits for both the ridges with a few outliers and even fewer ridges that we do not find at all. The main exceptions to this are at around redshift $z<0.05$ where the bins do not contain many galaxies and we end up with sparse ridges. For the redshift bins very close to us we cannot fit anything below redshift $z=0.021$ as the ridges are too irregular and the method can no longer fit them to satisfying precision. For the star-forming ridge we see a large increase in dispersion at higher redshift. This is mainly due to how sparse the ridge becomes at this point, making it harder to detect.

\subsection{Evolution of ridges in redshift}

By plotting each of our measured ridge SFRs, we can then see a relationship between the SFR and redshift (Fig. 5). It is obvious that the relationship is linear for the whole range of redshifts for the star-forming ridge and linear in about half the redshift range for the quenched ridge. It could be argued that a logarithmic fit would make a better fit for the quenched ridge but it seems more likely that there is another answer for the high redshift part of the quenched ridge, since the SFR values for the ridge stay almost constant in that part. This is likely due to a few different factors such as difficulty in observing the quenched ridge at this redshift or due to us using a shallower incline in the SFR $-M_{\star}$ plane than appropriate or that the ridges are not linear in the SFR- $M_{\star}$ plane but actually curve downwards at higher mass ranges as suggested by Schreiber et al. (2014) and Ciesla et al. (2017). This would lead to higher estimates as we go to higher $z$ since we only detect the high mass end of the ridge in that region. This fit does not seem to be widely accepted as people are still using linear ridges in some papers (Renzini \& Peng 2015 and Davies et al. 2016).

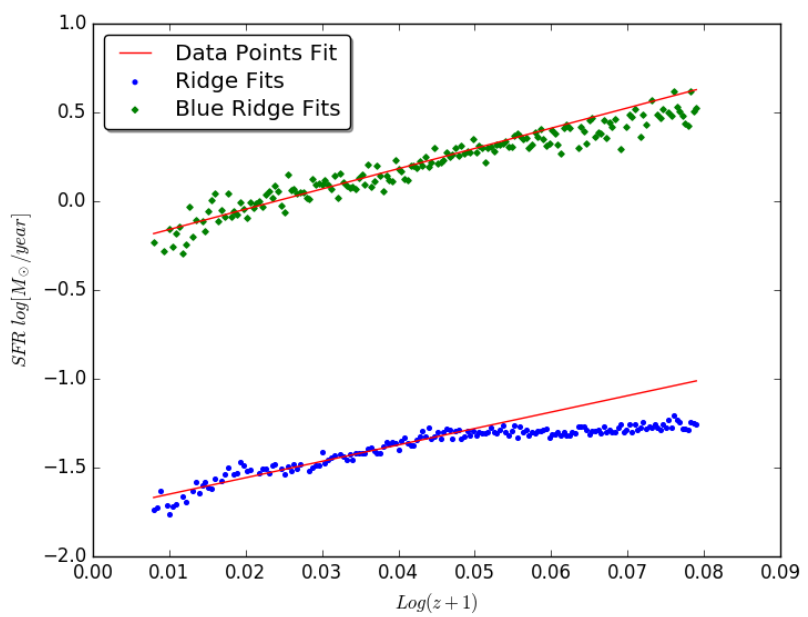

Fig. 5. Evolution of star-forming ridge and the quenched ridge SFR with redshift. For the star-forming ridge the best fit seems to be a linear one whereas for the quenched ridge it seems that above about redshift $\log (z+1)=0.055$ the measurements diverges from the linear fit. This could be due to a systematic error in the measurement of star formation rates at higher redshifts or due to the completeness test removing the left part of an already small quenched ridge.

\subsection{Comparison with observed clusters}

We can now apply the relationship between the measured ridge SFR and redshift to our galaxy cluster sample to find their redshifts. By using our method with the exact same parameters as when using bins, we find a few clusters where a ridge can be measured. The clusters are very close to the expected value in SFR compared to their known redshift (see Fig. 6), with all of them coming within $1 \sigma$ or very close to it. We even find a measurement for the Virgo cluster despite being unable to get a measurement in the redshift bin containing the cluster. This is due to the Virgo cluster not being messy and because the requirements for the redshift bins are harsher than for the clusters. Unfortunately when converting the uncertainty to redshift, it ends up being a large range of redshifts and becomes useless in determining exact an redshift compared to other, more precise, methods. The main cause of this appears to be the number of galaxies per cluster. To obtain reliable results, this method requires at least 100 members per cluster. Due to the low member count of many of the clusters this means that even in the best cases the results from the clusters are only as precise as the worst cases for the redshift bins, with the clusters typically having $>200 \%$ higher variance in their measurement.

It should be noted that a lot of clusters in our sample have fewer than 100 members with masses above $10^{10} M_{\odot}$. The number decreases further since we also need the SFR of the galaxy, which has not been reliably measured in all cases. For these cases we can still measure the ridges but the measurement becomes dominated by the spread of galaxies, which theoretically should yield the double ridge structure we expect, but due to the low number of galaxies we often see irregular ridges or overdensities in the SFR $-M_{\star}$ plane that thwart the fits.

\subsection{Limits}

From our experiments, there are clear requirements on the data for our approach to work reliably. The first is that the objects we are measuring have to be at least at a redshift $z>0.02$. If it is less than this the ridge will become too irregular causing the fit 


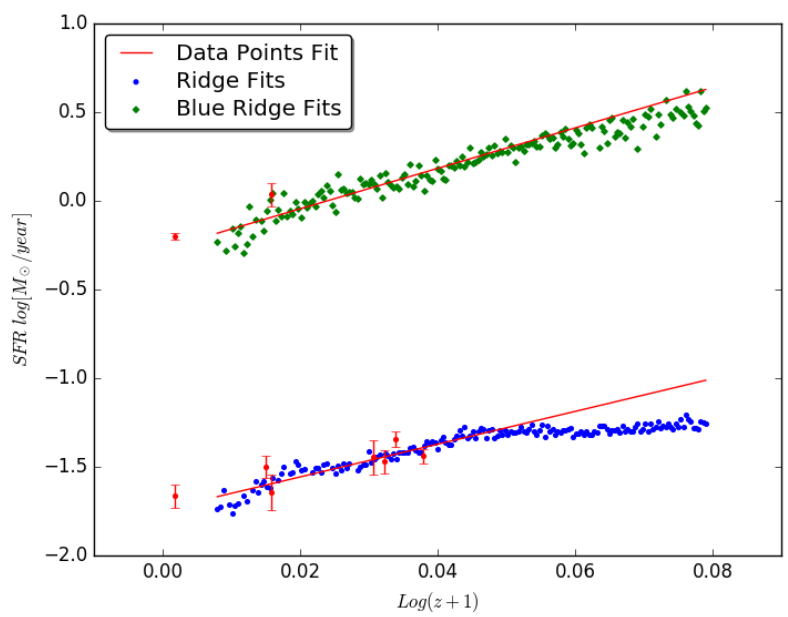

Fig. 6. Comparison of our fits to the redshift bin data and fits to a sample of galaxy clusters. They are all within our expectation; our fit for the Virgo cluster is sufficiently close that it even extends to lower redshifts beyond what we can do with the redshift bins.

to become inaccurate. There is a similar limit at higher $\mathrm{z}$ due to the incompleteness of data.

Applying the method to clusters, we see that we can use clusters with more than 100 galaxies to get good results (standard deviation of $\sigma_{\mathrm{SFR}}<0.1 \log \left(M_{\odot}\right.$ year $\left.^{-1}\right)$. For the quenched ridge the resulting redshift measurements have an uncertainty of $\sigma_{z}= \pm 0.024 \cdot(z+1)$ in our worst case but average to $\sigma_{z}=$ $\pm 0.017 \cdot(z+1)$ for all cases and go as low as $\sigma_{z}= \pm 0.01 \cdot(z+1)$ in the best case. For the star-forming ridge we only find two clusters that satisfy our requirements and they have a standard deviation of $\sigma_{z}= \pm 0.004 \cdot(z+1)$ and $\sigma_{z}= \pm 0.013 \cdot(z+1)$. To calculate these standard deviations, we have not taken the cluster's redshift into account as we have not been able to get enough galaxy cluster measurements to verify if there is a trend relating uncertainty to redshift. Assuming that this accuracy is adequate the method can find proper redshifts to within that accuracy with $>100$ galaxy members in the cluster. The same limits for the redshift bins also apply to the clusters but the number of member galaxies is the primary difference, making it much easier to get measurements on the bins in most cases.

\subsection{Comparison to others methods}

The analysis that we have presented in this work uses spectroscopy to obtain the critical parameters required to populate the galaxy main sequence plane (star formation rates and stellar masses). Naturally, one could simply apply one of a number of techniques to these spectra to obtain the overall recession velocity of the galaxy cluster itself (e.g. Yahil \& Vidal 1977; Zabludoff et al. 1990; Beers et al. 1990; Carlberg et al. 1996; Diaferio 1999). From this point of view, the method presented here should be viewed as an additional way in which the redshift of clusters could be obtained, and not necessarily the easiest or most efficient. However, it is possible to populate the galaxy main sequence plane in different manners, and hence finding the redshift of a galaxy cluster from the main sequence plane alone may be more expedient than undertaking observationally expensive (in comparison to imaging) spectroscopy. For the stellar mass axis, there are a number of authors in the literature advancing methods to compute galaxy stellar mass based on photometric observation alone. For example, Hsieh \& Yee
(2014) introduce a direct empirical photometric method that can compute stellar mass from a small number of passbands (see also Budavari et al. 2009); Brinchmann \& Ellis (2000) map out the relationship between stellar mass and $K$-band luminosity; others use multi-wavelength broadband photometry to derive very reasonable estimates for stellar masses in their own surveys (for example Taylor et al. 2011; Zibetti et al. 2009; Dye et al. 2008; Bell et al. 2003). However these methods should be used with care at high redshifts where a variety of uncertainties could affect the results (cf. Mitchell et al. 2013). For the star formation rate axis, spectral energy distribution (SED) modelling can potentially yield the required parameter. For example, Flores et al. (1999) use radio, mid- and near-IR, optical, and UV photometry to extrapolate the far-IR luminosity and thus estimate SFRs that are not extinction dependant. The use of SED modelling for photometry has also been applied to other studies to yield SFR, including but not limited to Maraston et al. (2010), Yuan et al. (2012), and Straatman et al. (2016); other methods such as machine learning are yielding very promising results in this area as well (Stensbo-Smidt et al. 2016).

\section{Conclusion}

The method succeeds in finding clear ridges in most of the redshift bins and we were able to determine a clear correlation between the ridge SFR and redshift. We then used the same method to find the redshift for several clusters and were able to find the ridge and get a good measurement of redshift for seven clusters. When comparing these results from the clusters to the fit from the redshift bins. we see that the inclination of the fit is very shallow so the error from the cluster measurements results in a somewhat large range of redshift, with uncertainties $\sigma_{z}= \pm 0.017 \cdot(z+1)$. We do not detect any correlation between outliers and any measurable parameter of the clusters such as luminosity or substructuring, suggesting that this dispersion is largely due to lack of data.

Therefore, this method can only be applied to those clusters with many members (each with SFR and stellar mass well determined) to yield a redshift value with a low standard deviation. Even then, the scatter present in our method means that such a redshift must be regarded as a secondary method at best.

Acknowledgements. MOL and KAP gratefully acknowledge funding from the University of Hull through the "Origins Cluster" (2016). MOL and KAP acknowledge the support of STFC through the University of Hull Consolidated Grant ST/R000840/1 This research has made use of the X-Ray Clusters Database (BAX), which is operated by the Laboratoire d'Astrophysique de Tarbes-Toulouse (LATT), under contract with the Centre National d'Etudes Spatiales (CNES). Funding for SDSS-III has been provided by the Alfred P. Sloan Foundation, the Participating Institutions, the National Science Foundation, and the US Department of Energy Office of Science. The SDSSIII web site is http://www.sdss3.org/. SDSS-III is managed by the Astrophysical Research Consortium for the Participating Institutions of the SDSS-III Collaboration including the University of Arizona, the Brazilian Participation Group, Brookhaven National Laboratory, Carnegie Mellon University, University of Florida, the French Participation Group, the German Participation Group, Harvard University, the Instituto de Astrofisica de Canarias, the Michigan State/Notre Dame/JINA Participation Group, Johns Hopkins University, Lawrence Berkeley National Laboratory, Max Planck Institute for Astrophysics, Max Planck Institute for Extraterrestrial Physics, New Mexico State University, New York University, Ohio State University, Pennsylvania State University, University of Portsmouth, Princeton University, the Spanish Participation Group, University of Tokyo, University of Utah, Vanderbilt University, University of Virginia, University of Washington, and Yale University. We also thank the anonymous referee whose feedback has helped improve this paper. 


\section{References}

Beers, T. C., Flynn, K., \& Gebhardt, K. 1990, AJ, 100, 32

Bell, E. F., McIntosh, D. H., Katz, N., \& Weinberg, M. D. 2003, ApJS, 149, 289

Bell, E. F., Papovich, C., Wolf, C., et al. 2005, ApJ, 625, 23

Bouche, N., Dekel, A., Genzel, R., et al. 2010, ApJ, 718, 1001

Brinchmann, J., \& Ellis, R. 2000, ApJ, 536, L77

Brinchmann, J., Charlot, S., White, S. D. M., et al. 2004, MNRAS, 351, 1151

Budavari, T., Heinis, S., Szalay, A. S., et al. 2009, ApJ, 694, 1281

Bundy, K., Ellis, R. S., Conselice, C. J., et al. 2006, ApJ, 651, 120

Carlberg, R. G., Yee, H. K. C., Ellingson, E., et al. 1996, ApJ, 462, 32

Ciesla, L., Elbaz, D., \& Fensch, J. 2017, A\&A, 608, A41

Cowie, L. L., Songaila, A., Hu, E. M., \& Cohen, J. G. 1996, AJ, 112, 839

Daddi, E., Alexander, D. M., Dickinson, M., et al. 2007, ApJ, 670, 173

Davies, L. J. M., Driver, S. P., Robotham, A. S. G., et al. 2016, MNRAS, 461, 458

Davis, M., Guhathakurta, P., Konidaris, N. P., et al. 2007, ApJ, 660, L1

Diaferio, A. 1999, MNRAS, 309, 610

Dutton, A. A., van den Bosch, F. C., \& Dekel, A. 2010, MNRAS, 405, 1690

Dye, S., Eales, S. A., Aretxaga, I., et al. 2008, MNRAS, 386, 1107

Elbaz, D., Daddi, E., Borgne, D. L., et al. 2007, A\&A, 468, 33

Elbaz, D., Dickinson, M., Hwang, H. S., et al. 2011, A\&A, 533, A119

Eliche-Moral, M. C., Prieto, M., Gallego, J., \& Zamorano, J. 2010, A\&A, 519, A55

Flores, H., Hammer, F., Thuan, T. X., et al. 1999, ApJ, 517, 148

Fontanot, F., Lucia, G. D., Monaco, P., Somerville, R. S., \& Santini, P. 2009, MNRAS, 397, 1776

Gilbank, D. G., Bower, R. G., Glazebrook, K., et al. 2011, MNRAS, 414, 304

Guo, K., Zheng, X. Z., \& Fu, H. 2013, ApJ, 778, 23

Guo, K., Zheng, X. Z., Wang, T., \& Fu, H. 2015, ApJ, 808, L49

Guzmán, R., Gallego, J., Koo, D. C., et al. 1997, ApJ, 489, 559

Heavens, A., Panter, B., Jimenez, R., \& Dunlop, J. 2004, Nature, 428, 625

Hsieh, B. C., \& Yee, H. K. C. 2014, ApJ, 792, 102

Karim, A., Schinnerer, E., Martìnez-Sansigre, A., et al. 2011, ApJ, 730, 61
Kauffmann, G., White, S. D. M., Heckman, T. M., et al. 2004, MNRAS, 353, 713

Maraston, C., Pforr, J., Renzini, A., et al. 2010, MNRAS, 407, 830

Mitchell, P. D., Lacey, C. G., Baugh, C. M., \& Cole, S. 2013, MNRAS, 435, 87

Noeske, K. G., Faber, S. M., Weiner, B. J., et al. 2007a, ApJ, 660, L47

Noeske, K. G., Weiner, B. J., Faber, S. M., et al. 2007b, ApJ, 660, L43

Obreja, A., Brook, C. B., Stinson, G., et al. 2014, MNRAS, 442, 1794

Pannella, M., Elbaz, D., Daddi, E., et al. 2015, ApJ, 807, 141

Pimbblet, K. A., Penny, S. J., \& Davies, R. L. 2014, MNRAS, 438, 3049

Reddy, N. A., Steidel, C. C., Fadda, D., et al. 2006, ApJ, 644, 792

Renzini, A., \& Peng, Y. 2015, ApJ, 801, L29

Rodighiero, G., Daddi, E., Baronchelli, I., et al. 2011, ApJ, 739, L40

Rodighiero, G., Renzini, A., Daddi, E., et al. 2014, MNRAS, 443, 19

Salim, S., Rich, R. M., Charlot, S., et al. 2007, ApJS, 173, 267

Salmi, F., Daddi, E., Elbaz, D., et al. 2012, ApJ, 754, L14

Schreiber, C., Pannella, M., Elbaz, D., et al. 2014, A\&A, 575, A74

Sparre, M., Hayward, C. C., Feldmann, R., et al. 2016, MNRAS, 466, 88

Speagle, J. S., Steinhardt, C. L., Capak, P. L., \& Silverman, J. D. 2014, ApJS, 214,15

Spergel, D. N., Bean, R., Dore, O., et al. 2007, ApJS, 170, 377

Stensbo-Smidt, K., Gieseke, F., Igel, C., Zirm, A., \& Pedersen, K. S. 2016, MNRAS, 464, 2577

Straatman, C. M. S., Spitler, L. R., Quadri, R. F., et al. 2016, ApJ, 830, 51

Tasca, L. A. M., Fevre, O. L., Hathi, N. P., et al. 2015, A\&A, 581, A54

Taylor, E. N., Hopkins, A. M., Baldry, I. K., et al. 2011, MNRAS, 418, 1587

Thomas, D., Maraston, C., Bender, R., \& de Oliveira, C. M. 2005, ApJ, 621, 673

Weiner, B. J., Papovich, C., Bundy, K., et al. 2007, ApJ, 660, L39

Whitaker, K. E., van Dokkum, P. G., Brammer, G., \& Franx, M. 2012, ApJ, 754, L29

Wuyts, S., Schreiber, N. M. F., van der Wel, A., et al. 2011, ApJ, 742, 96

Yahil, A., \& Vidal, N. V. 1977, ApJ, 214, 347

Yuan, T.-T., Kewley, L. J., Swinbank, A. M., \& Richard, J. 2012, ApJ, 759, 66

Zabludoff, A. I., Huchra, J. P., \& Geller, M. J. 1990, ApJS, 74, 1

Zamojski, M. A., Schiminovich, D., Rich, R. M., et al. 2007, ApJS, 172, 468

Zibetti, S., Charlot, S., \& Rix, H.-W. 2009, MNRAS, 400, 1181 